\begin{tabular}{|c|l|}
\hline Title & Ballistic spin interferometer using the Rashba effect \\
\hline Author(s) & Koga, Takaaki; Nitta, Junsaku; Veenhuizen, Marc van \\
\hline Citation & $\begin{array}{l}\text { Physical Review B, 70(161302(R)), 161302-1-161302-4 } \\
\text { https://doi.org/10.1103/PhysRevB.70.161302 }\end{array}$ \\
\hline Issue Date & 2004 \\
\hline Doc URL & http://hdl.handle.net/2115/14680 \\
\hline Rights & Copyright $\odot 2004$ A merican Physical Society \\
\hline Type & article \\
\hline File Information & PRB70-16.pdf \\
\hline
\end{tabular}

Instructions for use 


\title{
Ballistic spin interferometer using the Rashba effect
}

\author{
Takaaki Koga, ${ }^{1,2, *}$ Junsaku Nitta, $^{2}$ and Marc van Veenhuizen ${ }^{2}$ \\ ${ }^{1}$ PRESTO, Japan Science and Technology Agency, 4-1-8 Honcho, Kawaguchi, Saitama, 332-0012, Japan \\ ${ }^{2}$ NTT Basic Research Laboratories, NTT Corporation, Atsugi, Kanagawa, 243-0198, Japan
}

(Received 22 June 2004; published 12 October 2004)

\begin{abstract}
We propose a ballistic spin interferometer using a square loop (SL) geometry, where an incident electron wave packet is split into a pair of partial waves by a "hypothetical" beam splitter. These electron partial waves, then, follow the SL path in the clockwise and counterclockwise directions, respectively, so that they interfere with each other at the incident point, retaining the spin degree of freedom. We find that the backscattering probability of an incident electron can be largely modulated by varying the magnitude of the Rashba spin-orbit coupling constant $\alpha$. We propose to make the proposed spin interferometry experiment using an artificial nanostructure fabricated in, for example, $\mathrm{In}_{0.52} \mathrm{Al}_{0.48} \mathrm{As} / \mathrm{In}_{0.53} \mathrm{Ga}_{0.47} \mathrm{As} / \mathrm{In}_{0.52} \mathrm{Al}_{0.48} \mathrm{As}$ quantum wells.
\end{abstract}

DOI: 10.1103/PhysRevB.70.161302

PACS number(s): 73.20.Fz, 73.23.Ad, 73.63.Hs, 71.70.Ej

Exploitation of spin degree of freedom for the conduction carriers provides a key strategy for finding new functional devices in semiconductor spintronics. ${ }^{1-4}$ A promising approach for manipulating spins in semiconductor nanostructures is the utilization of spin-orbit (s.o.) interactions. In this regard, lifting of the spin degeneracy in the conduction (or valence) band due to the structural inversion asymmetry is especially called "Rashba effect," ",6 the magnitude of which can be controlled by the applied gate voltages and/or specific sample design of the heterostructure. ${ }^{7,8}$

In the present work, we propose a ballistic spin interferometer (SI) using a square loop (SL) geometry, where an electron spin rotates by an angle $\theta$ due to the Rashba effect as it travels along a side of the SL ballistically. The use of the SL geometry for a SI is contrasted with our previous proposal of a SI using a ring (circular) geometry. ${ }^{4}$ There, we assumed that electrons in the interferometer travel along the curvature of the ring ballistically as well as adiabatically (without scattering), retaining both the dynamical spin phase and the geometrical Berry phase. In reality, experimental verification of such a SI has not been an easy task, ${ }^{9-11}$ because (1) electrons have to travel along the whole circle of the ring without scattering in order for us to see the predicted spin interference effect and (2) the universal conductance fluctuation (UCF), ${ }^{12}$ which is caused by the presence of randomly distributed elastic scatterers in the ring, would cover the signal of the spin interference. It is expected that these hurdles found in a SI using a circular loop would be circumvented in a SI using SL's. In a SL geometry, (1) electrons are required to travel ballistically only along sides of the SL. Electrons would, then, experience spin-preserving scattering (reflection) at each corner of the SL. This makes the consideration of the geometrical Berry phase unnecessary, because the spin preserving reflection of electrons is a nonadiabatic process, which simplifies the model calculation. (2) Other advantages of using SL's include the geometrical feasibility of two-dimensional arraying for diminishing the effects of the UCF and the Aharonov-Bohm (AB) oscillation ${ }^{13}$ as we discuss later.

An electronic band structure for a two-dimensional electron gas (2DEG) that is confined in an potential well sufficiently asymmetric to induce the Rashba effect has a spin splitting in the conduction band. We are aware of another mechanism that induces spin splittings due to the bulk inversion asymmetry, so-called "Dresselhaus term,"14 which we leave as a future research topic. The energy dispersion relation for a system with the Rashba effect is given by $E$ $=\hbar^{2} k^{2} / 2 m^{*} \pm \alpha k$, where $\alpha$ is the Rashba spin-orbit coupling constant and + and - signs are for spin up and down states, respectively, for a given wave vector $\mathbf{k}$. For example, assuming $\mathbf{k} \| \hat{\boldsymbol{x}}$ and taking the spin basis along $z$ axis (perpendicular to the 2DEG plane), we can write these two spin states as

$$
\boldsymbol{\Psi}_{\mathbf{k} \uparrow} \equiv \frac{1}{2}\left(\begin{array}{c}
1-i \\
1+i
\end{array}\right) e^{i k x}
$$

and

$$
\boldsymbol{\Psi}_{\mathbf{k} \downarrow} \equiv \frac{1}{2}\left(\begin{array}{c}
1+i \\
1-i
\end{array}\right) e^{i k x}
$$

respectively (spin pointing in the $\pm y$ direction), in the planewave approximation.

Let us first consider the mechanism of spin precession for $\mathbf{k} \| \hat{\boldsymbol{x}}$. In this case, wave vectors for the spin-up and -down states at a given energy (e.g., Fermi energy) are written as $\mathbf{k}=(k \mp \Delta k, 0,0)$, where - and + signs correspond to spin-up and -down states as defined above, respectively, and $\Delta k$ $=m^{*} \alpha / \hbar^{2}+O\left(\alpha^{2}\right)$. Making a linear combination between these states, one can construct a following spin coherent state:

$$
\begin{aligned}
\boldsymbol{\Psi}_{\mathbf{k}} & \equiv \frac{1}{\sqrt{2}}\left(\boldsymbol{\Psi}_{\mathbf{k}-\Delta \mathbf{k} \uparrow}+\boldsymbol{\Psi}_{\mathbf{k}+\Delta \mathbf{k} \downarrow}\right) \\
& =\frac{1}{2 \sqrt{2}}\left\{\left(\begin{array}{c}
1-i \\
1+i
\end{array}\right) e^{i(k-\Delta k) x}+\left(\begin{array}{c}
1+i \\
1-i
\end{array}\right) e^{i(k+\Delta k) x}\right\} \\
& =e^{i k x} \frac{1}{\sqrt{2}}\left(\begin{array}{c}
\cos (\Delta k x)-\sin (\Delta k x) \\
\cos (\Delta k x)+\sin (\Delta k x)
\end{array}\right)
\end{aligned}
$$

We see that $\boldsymbol{\Psi}_{\mathbf{k}}$ can be written as a product between the plane-wave part $e^{i k x}$ and the spin state vector part 


$$
\frac{1}{\sqrt{2}}\left(\begin{array}{c}
\cos (\Delta k x)-\sin (\Delta k x) \\
\cos (\Delta k x)+\sin (\Delta k x)
\end{array}\right)
$$

which exemplifies the motion of spin precession as $\boldsymbol{\Psi}_{\mathbf{k}}$ propagates along $x$ axis:

$$
\begin{gathered}
{\left[\begin{array}{c}
\frac{1}{\sqrt{2}}\left(\begin{array}{l}
1 \\
1
\end{array}\right) \\
+x \text { direction } \\
(\Delta k x=0)
\end{array}\right]} \\
\rightarrow \quad\left[\begin{array}{c}
\text { polarized in the } \\
-z \text { direction } \\
(\Delta k x=\pi / 4)
\end{array}\right] \\
{\left[\begin{array}{c}
\text { polarized in the } \\
-x \text { direction } \\
(\Delta k x=\pi / 2)
\end{array}\right] \text { and so on. }}
\end{gathered}
$$

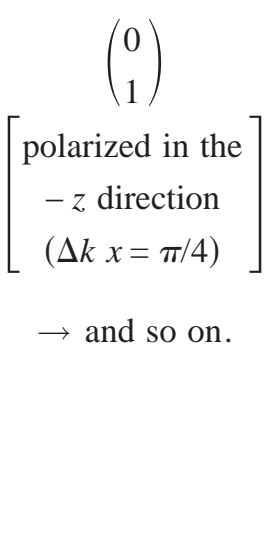

The proposed SI in the present work is illustrated in Fig. 1 , where three totally reflective mirrors are placed at three corners of the SL and a beam splitter, the property of which is defined below, is placed at the fourth corner. We also apply an external magnetic field $B$ in the $z$ direction. In this SI, an incident electron is injected to the beam splitter from path 1 . The injected wave packet is, then, split into two equally weighted partial waves $\boldsymbol{\Psi}_{1}$ and $\boldsymbol{\Psi}_{2}$, where $\boldsymbol{\Psi}_{1}$ follows path 1 (toward the $+y$ direction) and $\boldsymbol{\Psi}_{2}$ follows path 2 (toward $+x$ direction). We note that any junction with more than two leads attached to it would do the job of the beam splitter experimentally, since an electron wave injected from a lead to the junction would be split into multiple partial waves that propagate through the other leads attached. Thus, explicit introduction of the beam splitter is unnecessary experimentally. Similarly, any junction with two leads attached would do the job of the reflective mirrors, since electrons in a lead would be led to the other lead through the junction. The specularity of the mirrors should be optimized experimentally.

The quantum mechanical state of the incident electron (before passing through the beam splitter) can be written as

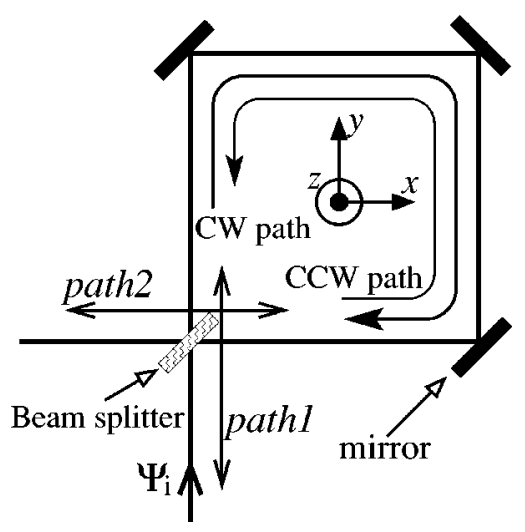

FIG. 1. Schematic diagram for the proposed spin interferometer in a square loop geometry. An incident wave $\boldsymbol{\Psi}_{i}$ is split by the beam splitter at the lower left corner.

$$
\boldsymbol{\Psi}_{\mathbf{i}}=\left(\begin{array}{l}
\text { path 1 } \\
\text { path 2 }
\end{array}\right)=\left(\begin{array}{l}
\alpha \\
\beta
\end{array}\right),
$$

where $\left(\begin{array}{c}\alpha \\ \beta\end{array}\right)$ represents a general spin state vector $\left(\alpha \alpha^{*}+\beta \beta^{*}\right.$ $=1$ ) using the spin basis along $z$ axis. The property of the beam splitter introduced above is defined by the following unitary operator:

$$
U=\frac{1}{\sqrt{2}}\left(\begin{array}{cc}
\mathbf{I} & \mathbf{I} \\
\mathbf{I} & -\mathbf{I}
\end{array}\right),
$$

where $\mathbf{I}$ denotes the $2 \times 2$ identity matrix. Thus, we apply $\boldsymbol{\Psi}_{\boldsymbol{i}}$ to $U$,

$$
U \boldsymbol{\Psi}_{i}=\left(\begin{array}{c}
\frac{1}{\sqrt{2}}\left(\begin{array}{c}
\alpha \\
\beta
\end{array}\right) \\
\frac{1}{\sqrt{2}}\left(\begin{array}{l}
\alpha \\
\beta
\end{array}\right)
\end{array}\right) \equiv\left(\begin{array}{l}
\boldsymbol{\Psi}_{1} \\
\boldsymbol{\Psi}_{2}
\end{array}\right) .
$$

We note that partial waves $\boldsymbol{\Psi}_{1}$ and $\boldsymbol{\Psi}_{2}$, subsequently, propagate through the SL path in the clockwise (CW) and counterclockwise $(\mathrm{CCW})$ directions, respectively.

Now, we consider the interference between the partial waves $\boldsymbol{\Psi}_{1}$ and $\boldsymbol{\Psi}_{2}$ when they come back to the incident point after passing through the SL paths. It is noted that the planewave parts of the $\mathrm{CW}$ and $\mathrm{CCW}$ paths are related to each other by the time reversal symmetry, i.e., the time reversal path of the $\mathrm{CW}$ path is the regular $\mathrm{CCW}$ path, and vice versa. The interference of this type manifests itself in (1) the weak localization (or weak antilocalization) phenomena in diffusive conductors ${ }^{15}$ and (2) the magnetoresistance oscillation with a period corresponding to the magnetic flux half quanta $\Phi_{0}=h / 2 e$, denoted as the Al'tshuler-Aronov-Spivak (AAS) oscillation. ${ }^{16}$ From Eq. (1), we can see that both partial waves $\boldsymbol{\Psi}_{1}$ and $\boldsymbol{\Psi}_{2}$ acquire an equal phase factor $e^{i k 4 L}$ from the plane-wave part after passing through the SL path, where $L$ is the side length of the SL. Therefore, we can safely neglect the plane wave part of the wave function when discussing the spin interference effect in the present SI.

Let $\boldsymbol{\Psi}_{\mathrm{CW}}$ describe the electron spin that emerges on path 2 as a result that partial wave $\boldsymbol{\Psi}_{1}$ passes through the $\mathrm{CW}$ path:

$$
\begin{aligned}
\boldsymbol{\Psi}_{\mathrm{CW}} & =e^{i \phi / 2} \mathbf{R}_{-\hat{y}}(\theta) \mathbf{R}_{+\hat{x}}(\theta) \mathbf{R}_{+\hat{y}}(\theta) \mathbf{R}_{-\hat{x}}(\theta) \boldsymbol{\Psi}_{1} \\
& \equiv e^{i \phi / 2} \mathbf{A} \boldsymbol{\Psi}_{1} \rightarrow \text { path 2, }
\end{aligned}
$$

where $\mathbf{R}_{\hat{\boldsymbol{\xi}}}(\theta) \mathrm{s}(\hat{\boldsymbol{\xi}}= \pm \hat{\boldsymbol{x}}, \pm \hat{\boldsymbol{y}})$ denote quantum mechanical rotation operators for spin $1 / 2$ about $\hat{\boldsymbol{\xi}}$ direction by an angle $\theta$ applying the right-handed screw rule.

$$
\mathbf{R}_{\hat{\boldsymbol{\xi}}}(\theta)=\mathbf{I} \cos \frac{\theta}{2}-i \hat{\boldsymbol{\xi}} \cdot \boldsymbol{\sigma} \sin \frac{\theta}{2},
$$

where $\boldsymbol{\sigma}$ is the vector expression of the Pauli spin matrices. In Eqs. (5) and (6), $\theta$ is the spin precession angle that an incident spin pointing $+z$ direction would experience while traveling through a side of the square in the presence of the 
Rashba effect $(\theta=2 \Delta k L)$ and $e^{i \phi / 2}$ is the phase factor acquired from the vector potential that is responsible for the magnetic field $B(\mathbf{B} \| \hat{z})$ piercing the SL $\left(\phi=2 e B L^{2} / \hbar\right)$. In a similar way, $\boldsymbol{\Psi}_{\mathrm{CCW}}$ is the spin state vector describing the partial wave that emerges on path 1 as a result that partial wave $\boldsymbol{\Psi}_{2}$ passes through the CCW path:

$$
\boldsymbol{\Psi}_{\mathrm{CCW}}=e^{-i \phi / 2} \mathbf{A}^{-1} \boldsymbol{\Psi}_{2} \rightarrow \text { path } 1 .
$$

$\boldsymbol{\Psi}_{\mathrm{CW}}$ and $\boldsymbol{\Psi}_{\mathrm{CCW}}$ hit the beam splitter from path 2 and path 1 , respectively, and form the final (interfered) state for the electron wave,

$$
\begin{aligned}
\boldsymbol{\Psi}_{\text {final }} & \equiv\left(\begin{array}{l}
\boldsymbol{\Psi}_{1}^{\prime} \\
\boldsymbol{\Psi}_{2}^{\prime}
\end{array}\right)=U^{-1}\left(\begin{array}{c}
\boldsymbol{\Psi}_{\mathrm{CCW}} \\
\boldsymbol{\Psi}_{\mathrm{CW}}
\end{array}\right) \\
& =\frac{1}{\sqrt{2}}\left(\begin{array}{cc}
\mathbf{I} & \mathbf{I} \\
\mathbf{I} & -\mathbf{I}
\end{array}\right)\left(\begin{array}{c}
e^{-i \phi / 2} \mathbf{A}^{-1} \boldsymbol{\Psi}_{2} \\
e^{i \phi / 2} \mathbf{A} \boldsymbol{\Psi}_{1}
\end{array}\right),
\end{aligned}
$$

where we used the unitarity of $U$. We find,

$$
\begin{aligned}
\boldsymbol{\Psi}_{1}^{\prime} & =\frac{1}{2}\left[e^{-i \phi / 2} \mathbf{A}^{-1}+e^{i \phi / 2} \mathbf{A}\right]\left(\begin{array}{l}
\alpha \\
\beta
\end{array}\right) \\
& =\left(\begin{array}{c}
\left(X+Y \cos \frac{\theta}{2}\right) \alpha+(1-i) Y \sin \frac{\theta}{2} \beta \\
(1+i) Y \sin \frac{\theta}{2} \alpha+\left(X-Y \cos \frac{\theta}{2}\right) \beta
\end{array}\right),
\end{aligned}
$$

where $\quad X=\cos (\phi / 2)\left\{\cos ^{2}(\theta / 2)+\sin ^{2}(\theta / 2)\left[\cos ^{2}(\theta / 2)\right.\right.$ $\left.\left.-\sin ^{2}(\theta / 2)\right]\right\}$, and $Y=2 \sin (\phi / 2) \sin ^{2}(\theta / 2) \cos (\theta / 2)$. We note that the probability that an incident wave $\boldsymbol{\Psi}_{i}$ is backscattered to path 1 is given by the inner product $\left\langle\boldsymbol{\Psi}_{1}^{\prime} \mid \boldsymbol{\Psi}_{1}^{\prime}\right\rangle$, which is a function of the initial spin state $\left(\begin{array}{c}\alpha \\ \beta\end{array}\right)$. We consider the spin interference effect for the case that an incident electron is spin unpolarized. We let

$$
\left(\begin{array}{l}
\alpha \\
\beta
\end{array}\right)=\left(\begin{array}{c}
\cos \frac{\zeta}{2} e^{i \chi / 2} \\
\sin \frac{\zeta}{2} e^{-i \chi / 2}
\end{array}\right),
$$

and calculate the expectation value of $\left\langle\boldsymbol{\Psi}_{1}^{\prime} \mid \boldsymbol{\Psi}_{1}^{\prime}\right\rangle$ for an unpolarized incident spin using the following formula:

$$
\begin{aligned}
\overline{\langle\text { physical quantity }\rangle} \equiv & \frac{1}{4 \pi} \int_{0}^{\pi} \sin \zeta d \zeta \int_{0}^{2 \pi} d \chi \\
& \times\langle\text { physical quantity to be averaged }\rangle
\end{aligned}
$$

We shall see that $\left\langle\boldsymbol{\Psi}_{1}^{\prime} \mid \boldsymbol{\Psi}_{1}^{\prime}\right\rangle$ contains terms proportional to $|\alpha|^{2}-|\beta|^{2}, \alpha^{*} \beta$, and $\alpha \beta^{*}$. It is easily shown that the expectation values of these terms become zero for an unpolarized incident spin using Eq. (11) . We finally obtain

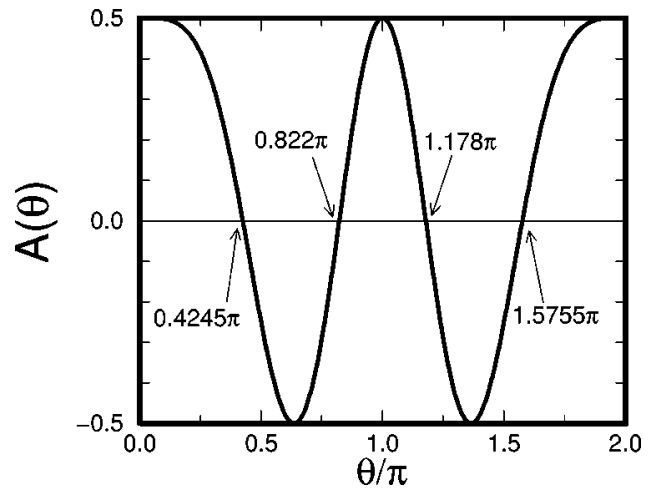

FIG. 2. Amplitude of the oscillatory part of the backscattering probability $\overline{\left\langle\boldsymbol{\Psi}_{1}^{\prime} \mid \boldsymbol{\Psi}_{1}^{\prime}\right\rangle}$ in the proposed spin interferometer as a function of the spin precession angle $\theta$ along a side of the square loop.

$$
\begin{aligned}
\overline{\left\langle\boldsymbol{\Psi}_{1}^{\prime} \mid \boldsymbol{\Psi}_{1}^{\prime}\right\rangle} & =X^{2}+\left(1+\sin ^{2} \frac{\theta}{2}\right) Y^{2} \\
& =\frac{1}{2}+\frac{1}{4}\left(\cos ^{4} \theta+4 \cos \theta \sin ^{2} \theta+\cos 2 \theta\right) \cos \phi \\
& \equiv \frac{1}{2}+A(\theta) \cos \phi .
\end{aligned}
$$

We note that the oscillation of $\overline{\left\langle\boldsymbol{\Psi}_{1}^{\prime} \mid \boldsymbol{\Psi}_{1}^{\prime}\right\rangle}$ as a function of $\phi$ corresponds to the AAS oscillation in mesoscopic physics, whose amplitude is given by $A(\theta)=\frac{1}{4}\left(\cos ^{4} \theta\right.$ $\left.+4 \cos \theta \sin ^{2} \theta+\cos 2 \theta\right)$ in the present calculation. We plot $A(\theta)$ as a function of $\theta$ in Fig. 2 .

Experimentally, we propose to fabricate an array of micron-sized SL's in $\mathrm{In}_{0.52} \mathrm{Al}_{0.48} \mathrm{As} / \mathrm{In}_{0.53} \mathrm{Ga}_{0.47} \mathrm{As} /$ $\mathrm{In}_{0.52} \mathrm{Al}_{0.48} \mathrm{As}$ quantum wells $(\mathrm{QW})$ using conventional nanolithographic techniques, where we investigate the amplitude of the AAS oscillation ${ }^{16}$ around $B=0$ as a function of the applied gate voltage. We illustrate an example of the SL array sample in Fig. 3, where electrons in the shaded region are depleted by the nano fabrication process. The undepleted white region consists of six SL's and every neighboring pair of the loops is electrically connected at the middle of the side. Note that the incident electrons are injected from the sides of the SLs in Fig. 3, not from the corners as we assumed in the theoretical model. In fact, our further theoretical investigation showed that the magnitude of $\overline{\left\langle\boldsymbol{\Psi}_{1}^{\prime} \mid \boldsymbol{\Psi}_{1}^{\prime}\right\rangle}$ does not depend on where to inject the incident electron in the perimeter of the SL. ${ }^{17}$ Therefore, the gate-voltage dependence of the AAS oscillation amplitude for a sample described by Fig. 3 should still be predicted by $A(\theta)$.

In an actual SL array sample, the length $L$ should ideally be smaller than the electron mean free path $l$, though it still has to be large enough for the spin precession angle $\theta$ to be gate-controllable to a significant extent. Values of $L$ about $1.5 \mu \mathrm{m}$ would satisfy these requirements at $T=0.3 \mathrm{~K}$. The width $W$ for the channel should be smaller than $L$, though, of which values should be optimized experimentally. In actual samples, the number of SL's that should be arrayed twodimensionally is much larger than six in order to diminish quantum fluctuations (QF's) like (1) the $\mathrm{AB}$ oscillation with 


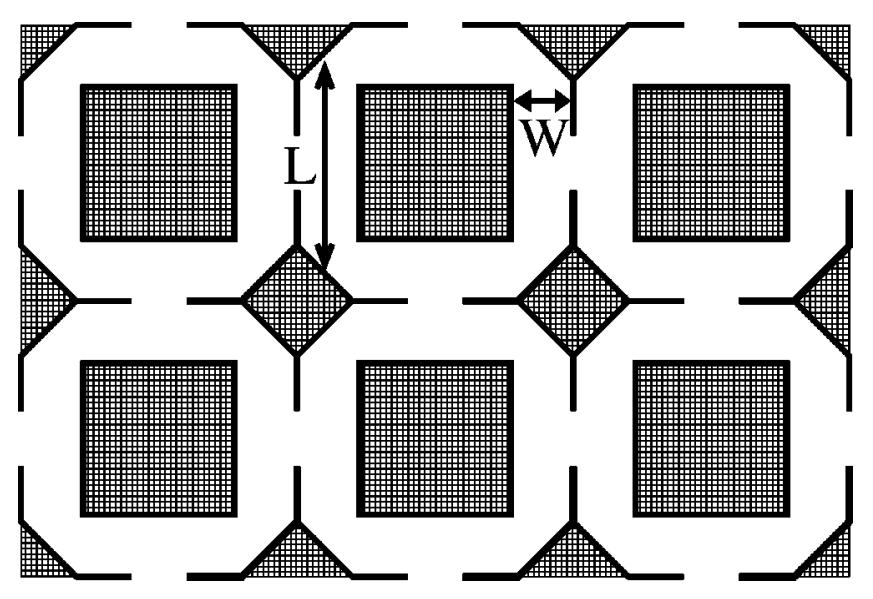

FIG. 3. An illustration of the array of nanolithographically defined square loops that we propose to use for testing the proposed spin interferometry experiment. Each square loop has its corners cut at an angle of $45^{\circ}$ to reflect the electrons coming from a side to the other side. The whole array is covered with a gate electrode for the control of the value of the Rashba constant $\alpha$ by the applied gate voltage. A typical length $L$ for the side of the square loop is $1.5 \mu \mathrm{m}$ and the size of the channel width $W$ should be optimized experimentally.

the period corresponding to the magnetic flux quanta $h / e$ and (2) the UCF. Both of these effects, which manifest themselves in a single-loop sample as QF's of the electric conductance as a function of $B$ and the applied gate voltage, respectively, are expected to be averaged out to zero by arraying a number of SL's. ${ }^{18}$ For the measurements, we pass an electric current across the whole array and measure the electric resistance there that is related to $\overline{\left\langle\boldsymbol{\Psi}_{1}^{\prime} \mid \boldsymbol{\Psi}_{1}^{\prime}\right\rangle}$ as a function of $B$ at temperatures typically lower than $0.3 \mathrm{~K}$.
In a QW system with the Rashba effect, spin precession angle $\theta$ is given by

$$
\theta=2 \Delta k L=\frac{2 \alpha m^{*}}{\hbar^{2}} L
$$

In the $\operatorname{In}_{0.52} \mathrm{Al}_{0.48} \mathrm{As} / \mathrm{In}_{0.53} \mathrm{Ga}_{0.47} \mathrm{As} / \mathrm{In}_{0.52} \mathrm{Al}_{0.48} \mathrm{As} \mathrm{QW}$ system, the magnitude of $|\alpha|$ is typically $2 \times 10^{-12} \mathrm{eV} \mathrm{m} .^{8}$ The variation of $\alpha$ value that can be achieved by the applied gate voltage is approximately $\pm 5 \times 10^{-13} \mathrm{eV} \mathrm{m}$. For example, for sample 3 in Ref. 8, using $m^{*}=0.047 m_{0}$ (the effective mass at Fermi energy) and $L=1.8 \mu \mathrm{m}, \theta$ can be varied from $0.29 \pi$ to $\pi$ (the corresponding $\alpha$ values are $0.4 \times 10^{-12} \mathrm{eV} \mathrm{m}$ and $1.4 \times 10^{-12} \mathrm{eV} \mathrm{m}$, respectively) by the applied gate voltage. With this variation of $\theta$, we see that the value of $A(\theta)$ is also varied from the minimum value $[A(\theta)=-0.5$ for $\theta=0.64 \pi]$ to the maximum value $[A(\theta)=0.5$ for $\theta=\pi]$. This result predicts that the amplitude of the AAS oscillation should be drastically modulated (sometimes even change signs) by the applied gate voltage in the actual experiments.

In summary, we propose a ballistic spin interferometer using a square loop (SL) geometry. In this model, a pair of electron partial waves is created by a "hypothetical" beam splitter at the incident point of the interferometer, where one partial wave follows the square path in the clockwise direction and the other does in the counterclockwise direction. These partial waves, then, meet each other again at the incident point, where the spin interference should take place. We propose to use a SL array fabricated in $\operatorname{In}_{0.52} \mathrm{Al}_{0.48} \mathrm{As}$ / $\mathrm{In}_{0.53} \mathrm{Ga}_{0.47} \mathrm{As} / \mathrm{In}_{0.52} \mathrm{Al}_{0.48} \mathrm{As}$ quantum wells to test the results of the present model calculation, where the $\alpha$ (Rashba constant) values in this material system have been already studied quantitatively using the weak antilocalization analysis. ${ }^{8}$
* Present address: Graduate School of Information Science and Technology, Hokkaido University, Sapporo, 060-0814, Japan. Electronic address: koga@ist.hokudai.ac.jp

${ }^{1}$ D. Awschalom, N. Samarth, and D. Loss, Semiconductor Spintronics and Quantum Computation (Springer-Verlag, Berlin, 2002).

${ }^{2}$ S. Datta and B. Das, Appl. Phys. Lett. 56, 665 (1990).

${ }^{3}$ T. Koga, J. Nitta, H. Takayanagi, and S. Datta, Phys. Rev. Lett. 88, 126601 (2002).

${ }^{4}$ J. Nitta, F. E. Meijer, and H. Takayanagi, Appl. Phys. Lett. 75, 695 (1999).

${ }^{5}$ E. I. Rashba, Fiz. Tverd. Tela (Leningrad) 2, 1224 (1960) [Sov. Phys. Solid State 2, 1109 (1960)].

${ }^{6}$ Y. A. Bychkov and E. I. Rashba, J. Phys. C 17, 6039 (1984).

${ }^{7}$ J. Nitta, T. Akazaki, H. Takayanagi, and T. Enoki, Phys. Rev. Lett. 78, 1335 (1997).

${ }^{8}$ T. Koga, J. Nitta, T. Akazaki, and H. Takayanagi, Phys. Rev. Lett.
89, 046801 (2002).

${ }^{9}$ J. Nitta, H. Takayanagi, and S. Calvet, Microelectron. Eng. 47, 85 (1999).

${ }^{10}$ J. Nitta, F. Meijer, Y. Narita, and H. Takayanagi, Physica E (Amsterdam) 6, 318 (2000).

${ }^{11}$ J. Nitta, T. Koga, and H. Takayanagi, Physica E (Amsterdam) 12, 753 (2002).

${ }^{12}$ P. A. Lee and A. D. Stone, Phys. Rev. Lett. 55, 1622 (1985).

${ }^{13}$ Y. Aharonov and D. Bohm, Phys. Rev. 115, 485 (1959).

${ }^{14}$ G. Dresselhaus, Phys. Rev. 100, 580 (1955).

${ }^{15}$ G. Bergmann, Phys. Rep. 107, 1 (1984).

${ }^{16}$ B. L. Al'tshuler, A. G. Aronov, and B. Z. Spivak, Pis'ma Zh. Eksp. Teor. Fiz. 33, 101 (1981) [JETP Lett. 33, 94 (1981)].

${ }^{17}$ M. van Veenhuizen (unpublished).

${ }^{18}$ G. J. Dolan, J. C. Licini, and D. J. Bishop, Phys. Rev. Lett. 56, 1493 (1986). 